Rev. SINAPSIS, Vol. 5, No 2, Diciembre 2014

ISSN $1390-7832$

\title{
Identificación del síndrome de burnout y factores relacionados con este fenómeno en los profesionales de enfermería
}

\section{Identificación del síndrome de burnout}

Tania Alcázar Pichucho. Mg Sc.

Ministerio de Salud Pública. Universidad Estatal del Sur de Manabí, Ecuador

Contacto: taniameralcazar@hotmail.es

Receptado: 06/03/2014

Aceptado: 04/10/2014

\section{Resumen}

El síndrome o desgaste profesional o quemarse por el trabajo (Burnout) se presenta como un bosquejo aclarativo de dificultades laborales en profesionales de salud específicamente en el personal de enfermería, los cuales se encuentran en constante peligro de experimentar el síndrome de burnout, un proceso que agota o cansa los recursos físicos y emocionales aquejando a los individuos responsables del cuidado directo del paciente. El estudio pretende aportar una serie de determinaciones de las variables cognitivo-actitudinal (baja realización en el trabajo), variables emocionales (agotamiento emocional) y variables aptitudinales (despersonalización) las cuales permitirán una aproximación al perfil de riesgo de esta profesión. Se derivan del estudio diferencias significativas en función del turno, edad, estado civil, categoría contractual, antigüedad, la función que desempeña, año de ingreso a la institución, servicio en que se encuentra desempeñando su trabajo y la existencia de un segundo empleo. Así mismo se analizaron las consecuencias de este síndrome como también los factores relacionados para su aparición, como respuesta al estrés laboral que determinan problemas de salud en los profesionales de enfermería.

Palabras claves: Enfermería, Cuidado directo, edad, peso, peligro

\section{Identification of burnout syndrome and factors related to this phenomenon nursing}

\begin{abstract}
Syndrome or professional or burn through labor (Burnout) wear is presented as an explanatory sketch of labor difficulties health professionals specifically nurses, which are in constant danger of experiencing burnout exhausting process or tiring physical and emotional resources to afflict individuals responsible for direct patient care. The study aims to provide a series of
\end{abstract}


Rev. SINAPSIS, Vol. 5, N² 2, Diciembre 2014

determinations of cognitive-attitudinal variables (low fulfillment at work), emotional variables (emotional exhaustion) and attitudinal variables (depersonalization) which allow an approximation risk profile of this profession al. They are derived from the study significant differences by shift, age, marital status, contractual category, seniority, role, year of admission to the institution, service which is handling his job and the existence of a second job. Also the consequences of this syndrome as well as factors related to its appearance in response to job stress that determine health problems nurses were analyzed.

Keywords: Nursing, direct care, age, weight, danger

\section{Introducción}

El término burnout, cuya traducción correspondería a "estar quemado por el trabajo", (Tous, 2005) fue introducido a mediados de los años setenta para dar una explicación al proceso negativo que sufren los profesionales en su labor, que se traduce en deterioro en la atención profesional a los usuarios de las organizaciones de servicios de salud, además se lo relaciona como un desgaste profesional iniciándose con la aparición de bajos sentimientos de realización personal en el trabajo y paralelamente alto agotamiento emocional. (De Pablo, 2007)

El síndrome de bornout se considera como la fase avanzada del estrés profesional combinado de variables físicas, psicológicas y sociales y se produce cuando se desequilibran las expectativas en el ámbito profesional (Cabrera, 2005) establecido como una respuesta al estrés laboral crónico que afecta a aquellas personas cuyo trabajo tiene como centro ayudar y apoyar a otros, integrado por actitudes y sentimientos negativos hacía con las personas que se trabaja y hacia el propio rol profesional, (Gi- Monte, 2013) así como por la vivencia de encontrase emocionalmente agotado

Maslash y Jackson especialistas americanas en Psicología de la salud, han definido el bournout como un síndrome de estrés crónico de aquellas profesiones de servicios que se caracterizan por una atención intensa y prolongada con personas que están en una situación de necesidad o de dependencia. (Leiter, 2005)

El avance tecnológico, la cultura de mejorar la calidad de vida laboral y la exigencia de productividad ha llevado a consecuencias de impacto en la salud física y psicológica de los trabajadores, según referencias emitidas por la Organización Mundial de la Salud (OMS) y la Organización Internacional del Trabajo (OIT). 
Rev. SINAPSIS, Vol. 5, N² 2, Diciembre 2014

ISSN $1390-7832$

Son muchos los factores que alteran el estado emocional de los profesionales de salud, en el personal de enfermería se determinan fundamentalmente tres factores como: cansancio emocional, despersonalización y falta de realización personal (Hernandez, 2003) los cuales pueden provocar alteraciones psicológicas demostrando sentimientos de no tener que ofrecer a los demás con poca relación de empatía.

Existen variables que determinan o condicionan estos momentos como son la sobrecarga de trabajo por escasez de personal, trabajo en turnos rotativos especialmente en el turno de la noche, trato con usuarios problemáticos, contacto directo con la enfermedad el dolor y la muerte, conflicto del rol profesional, falta de motivación, falta de especificidad de funciones, etc.

Por lo expuesto anteriormente se considera que el síndrome no solo afecta la parte física sino también lo psíquico y emocional del personal de salud lo que inicialmente provocaría trastornos laborales. (Noboa, 2005)

El presente estudio recoge el conocimiento generado por diversos autores, así como la información generada en varias investigaciones relacionadas con este tema y a la solución del mismo mediante la necesidad de preocuparse más de la calidad de vida laboral el cual repercute sobre la sociedad en general.

El objetivo de esta investigación fue identificar la existencia del "Síndrome de Burnout", y factores de riesgo de este fenómeno, mediante la obtención y análisis de datos primarios. Conocer y profundizar en el significado, causas, características, síntomas, consecuencias, variables, prevención, apoyo social y personalidad. Adicionalmente obtener datos que proporcionen a los directivos de la organización, la información necesaria para la toma de acciones y decisiones que ayuden a mejorar la calidad de vida de sus profesionales y por lo tanto mejorar los objetivos y resultados de su Institución.

La hipótesis a defender en la investigación fue: Los profesionales de enfermería del hospital básico de jipijapa están expuestos al burnout por presentar factores relacionados con este fenómeno.

Las hipótesis específicas fueron: El síndrome de burnout influenciara en el desempeño de las labores del personal de salud. El cuestionario breve de burnout contribuirá a evaluar al personal de enfermería del hospital de la zona de estudio. Explicar las características y consecuencias ayudara a definir y determinar el burnout en los profesionales de enfermería. 
Rev. SINAPSIS, Vol. 5, No 2, Diciembre 2014

ISSN $1390-7832$

\section{Metodología}

El presente estudio es descriptivo, correlacional y transversal. Es descriptivo puesto que mide los conceptos y variables relacionados con el problema de la investigación, utiliza el método de encuesta como recurso para obtener información aplicando un conjunto de cuestionarios aplicados a un grupo determinado de individuos, no se manipulan las variables del estudio, se plantea el objetivo de describir las características psicológicas de la muestra seleccionada; como se pretende establecer la relación entre variables, se trata de un método de encuesta de investigación.

Recurre a la relación lineal entre variables sin que se pretenda atribuir una relación causal, por lo tanto, se trata de una aproximación correlacional, pretende determinar si las variables que se investigan poseen factores en común. Se puede apelar a la identificación del coeficiente de correlacion en función a las características de los datos ya sean estos ordinales o nominales. Se trata de una investigación transversal porque se recoge la información en una muestra una sola vez y durante un periodo de corta duración.

\section{Desarrollo}

El síndrome de burnout en el contexto laboral se lo define como el síndrome de desgaste profesional se manifiesta como un deterioro en la atención al usuario y hacia el propio rol profesional ocurriendo con mayor frecuencia en los profesionales de la salud. (Maslach, 1982)

El burnout no es un proceso estático, sino continuo desarrollándose secuencialmente en cuanto a la aparición de rasgos y síntomas globales en diferentes etapas, (Alvarez L, 2000) el progreso del síndrome no es igual para todas las personas, de manera que no todos los profesionales que entran en un proceso de burnout siguen el mismo proceso evolutivo.

Desde una perspectiva psicosocial el síndrome de quemarse por el trabajo es un síndrome cuyos síntomas son bajos niveles de realización personal en el trabajo, altos niveles de agotamiento emocional y despersonalización.

La falta de realización personal en el trabajo se define como la tendencia de los profesionales a evaluarse negativamente y de forma especial esa evaluación negativa afecta la habilidad en la realización del trabajo y a la relación de las personas a las que atienden, los trabajadores se sienten descontentos consigo mismo e insatisfechos con sus resultados laborales. (De la Peña, 2002) 
Rev. SINAPSIS, Vol. 5, N² 2, Diciembre 2014

ISSN $1390-7832$

Por agotamiento emocional se entiende la situación en la que los trabajadores sienten que ya no pueden dar más de sí mismos a nivel afectivo. Es una situación de agotamiento de la energía o los recursos emocionales propios, una experiencia de estar emocionalmente agotado debido al contacto "diario" y manteniendo con personas a las que hay que atender como objeto de trabajo.

La despersonalización puede ser definida como el desarrollo de actitudes y sentimientos negativos, como el cinismo, hacia las personas destinatarias del trabajo. Estas personas son vistas por los profesionales de enfermería de forma deshumanizada debido a un endurecimiento afectivo, lo que conlleva que les culpen por sus problemas. (Albadalejo, 2004)

El síndrome de quemarse por el trabajo es una respuesta al estrés laboral crónico que aparece cuando fallan las estrategias de afrontamiento que habitualmente emplea al individuo para manejar los estresores laborales. (Escriba V, 2000)

Así, el síndrome de quemarse por el trabajo es considerado un paso intermedio en la relación estrés- consecuencias del estrés de forma que, si permanece a lo largo del tiempo el estrés laboral tendrá consecuencias nocivas para el individuo, (Lopez Sorianno, 2002) en forma de enfermedad o falta de salud con alteraciones psicosomáticas ( alteraciones cardiorrespiratorias, jaquecas, gastritis, y ulcera, dificultad para dormir, mareos y vértigos,etc) y para la organización ( deterioro del rendimiento o de la calidad asistencial o de servicio, absentismo, rotación no deseada, abandono,).

Los estresores laborales elicitaran una serie de estrategias de afrontamiento que, en el caso de los profesionales de enfermería, deben ser efectivas para manejar las respuestas al estrés, pero también han de ser eficaces para eliminar los estresores, dado que los sujetos deben tratar diariamente con esa fuente de estrés. (Gil Monte, 2000) Cuando las estrategias de afrontamiento empleadas inicialmente no resultan exitosas conllevan al fracaso profesional y fracaso de las relaciones interpersonales con los pacientes y con sus familiares. Por ello, la respuesta desarrollada son sentimientos de baja realización personal en el trabajo y agotamiento emocional. Ante estos sentimientos el sujeto desarrolla actitudes de despersonalización como nueva forma de afrontamiento.

Para el desarrollo de este síndrome influyen las características individuales. Según Maslach y Jackson el deseo de destacar y obtener poco realistas puede llevar a estados de ansiedad y frustración. La vida privada del trabajador también influye en el desarrollo del síndrome. Las personas más vulnerables al síndrome son las más sensibles al auto exigencia, con baja 
Rev. SINAPSIS, Vol. 5, No 2, Diciembre 2014

tolerancia al fracaso, perfeccionamiento, controladoras de todo; así, desarrollan el sentimiento de indispensabilidad laboral. (Bernal, 2002)

\section{Resultados}




\section{CANSANCIO EMOCIONAL \\ CUADRO $\mathbf{N}^{\circ} 1$}

¿Cuándo está en su trabajo se siente de mal humor?

\begin{tabular}{|c|c|c|c|c|c|c|c|c|c|c|}
\hline OPCIÓN & EMERGENCIA & $\%$ & $\begin{array}{l}\text { C. QUIRÚRGICO } \\
\text { YOBSTÉTRICO }\end{array}$ & \% & $\begin{array}{l}\text { CONSULTA } \\
\text { EXTERNA }\end{array}$ & \% & $\begin{array}{c}\text { CLÍNICA } \\
\text { Y } \\
\text { CIRUGIA }\end{array}$ & $\%$ & $\begin{array}{l}\text { GINECOLOGIAA } \\
\text { Y PEDIATRIAA }\end{array}$ & 96 \\
\hline Nunca & 10 & 1796 & 11 & $18 \%$ & 11 & 1896 & 12 & $20 \%$ & 11 & $18 \%$ \\
\hline Rara vea & 2 & 396 & 1 & 296 & o & 096 & 1 & 296 & 1 & 296 \\
\hline $\begin{array}{l}\text { Con } \\
\text { frecuencia }\end{array}$ & 0 & 096 & 0 & 096 & 0 & $0 \%$ & 0 & 096 & 0 & $0 \%$ \\
\hline Siempre & 0 & $0 \%$ & 0 & 096 & 0 & $0 \%$ & 0 & 096 & 0 & $0 \%$ \\
\hline TOTAL & 12 & 2096 & 12 & 2096 & 11 & 1896 & 13 & $22 \%$ & 12 & $20 \%$ \\
\hline
\end{tabular}

CRAFICSN No 1

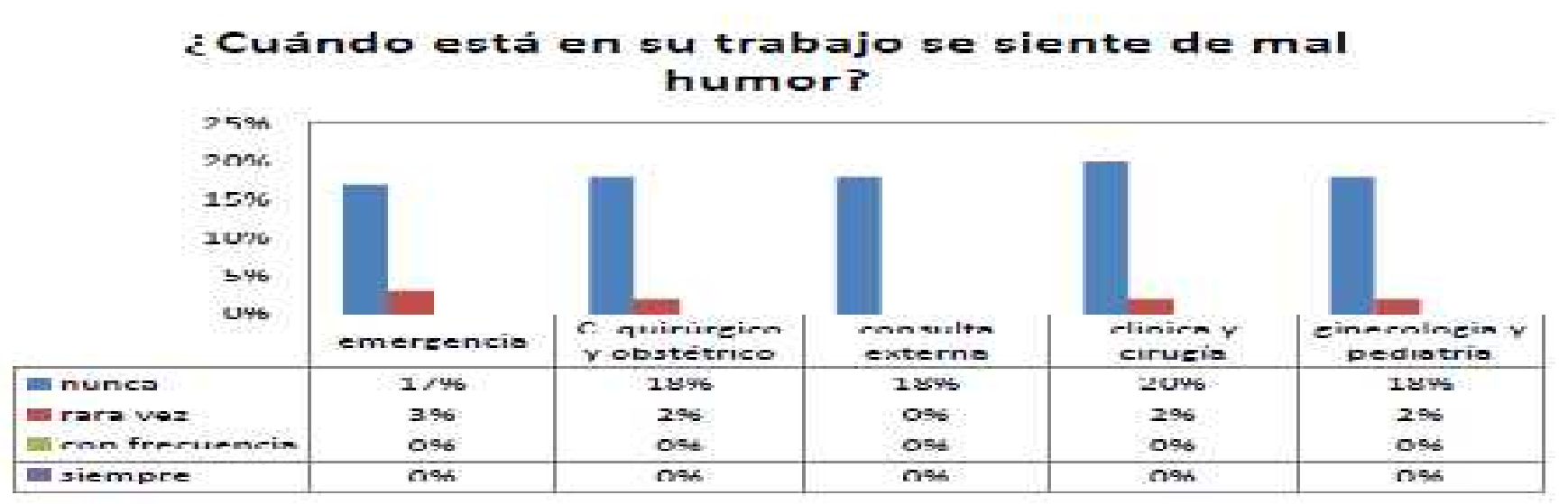

Fuente: Encuesta realizada a los profesionales de enfermería del Hospital Básico Jipijapa 


\section{DESPERSONALIZACIÓN \\ CUADRO $\mathrm{N}^{\circ} 2$}

¿Las personas a las que tiene que atender reconocen lo que hace Ud. por ellas?

\begin{tabular}{|c|c|c|c|c|c|c|c|c|c|c|}
\hline OPCIÓN & EMERGENCIA & $\%$ & $\begin{array}{c}\text { C. } \\
\text { QUIRGICO } \\
\text { Y } \\
\text { OBSTÉTRICO }\end{array}$ & $\%$ & $\begin{array}{c}\text { CONSULTA } \\
\text { EXTERNA }\end{array}$ & $\%$ & $\begin{array}{c}\text { CLÍNICA } \\
\text { Y } \\
\text { CIRUGIA }\end{array}$ & $\%$ & $\begin{array}{c}\text { GINECOLOGÍA } \\
\text { Y PEDIATRIA }\end{array}$ & $\%$ \\
\hline Nunca & 0 & $0 \%$ & 0 & $0 \%$ & 0 & $0 \%$ & 0 & $0 \%$ & 0 & $0 \%$ \\
\hline rara vez & 2 & $3 \%$ & 1 & $2 \%$ & 2 & $3 \%$ & 3 & $5 \%$ & 3 & $5 \%$ \\
\hline $\begin{array}{c}\text { con } \\
\text { frecuencia }\end{array}$ & 8 & $14 \%$ & 7 & $12 \%$ & 3 & $5 \%$ & 6 & $10 \%$ & 6 & $10 \%$ \\
\hline Siempre & 2 & $3 \%$ & 4 & $7 \%$ & 6 & $10 \%$ & 4 & $7 \%$ & 3 & $5 \%$ \\
\hline TOTAL & 12 & $20 \%$ & 12 & $20 \%$ & 11 & $18 \%$ & 13 & $22 \%$ & 12 & $20 \%$ \\
\hline
\end{tabular}

\section{GRÁFICO $\mathbf{N}^{\circ} 2$}

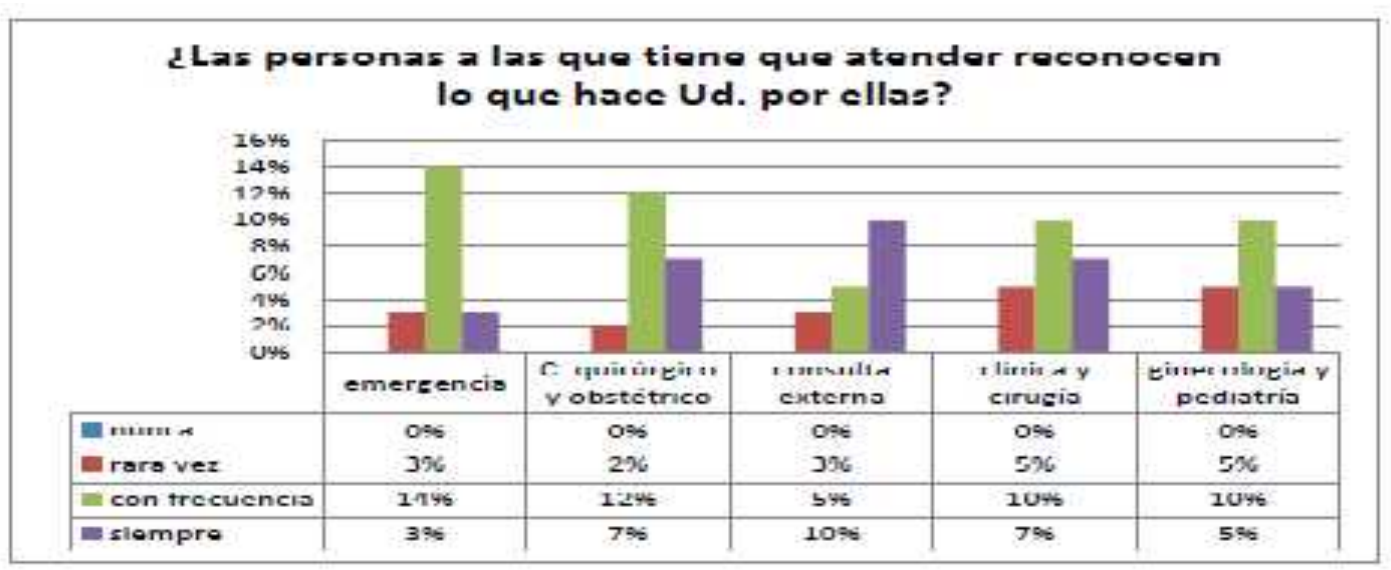

Fuente: Encuesta realizada a los profesionales de enfermería del Hospital Básico Jipijapa 


\section{REALIZACION PROFESIONAL}

CUADRO $\mathbf{N}^{\circ} 3$

¿Su interés por el desarrollo profesional es actualmente muy escaso?

\begin{tabular}{|c|c|c|c|c|c|c|c|c|c|c|}
\hline 9 & EMERGENCIA & $*$ & $\begin{array}{c}\text { C. } \\
\text { QUREURGICO Y } \\
\text { ORSTKTRICO }\end{array}$ & $\%$ & $\begin{array}{l}\text { CONSUITA } \\
\text { CXTERNA }\end{array}$ & \% & $\begin{array}{l}\text { clínicar } \\
\text { cinugia }\end{array}$ & * & $\begin{array}{c}\text { GIMECOLOGIAY } \\
\text { PCDIATILA }\end{array}$ & $\%$ \\
\hline vunca & 12 & 2006 & 12 & $20 \%$ & 11 & 1896 & 13 & $22 \%$ & 12 & 2046 \\
\hline ara vez & 0 & $0 \%$ & 0 & का & 0 & $0 x$ & 0 & $0 x$ & c & $\mathrm{CH}$ \\
\hline $\begin{array}{l}\text { zon } \\
\text { frcaucncia }\end{array}$ & 0 & $0 \%$ & 0 & $\cos$ & 0 & $0 *$ & 0 & $0 \%$ & c & $\mathrm{CS}$ \\
\hline Sieriple & u & U\% & $\bar{v}$ & Un & v & U* & 0 & $0 \%$ & 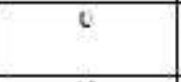 & $\mathrm{Cr}$ \\
\hline TOTAL & $\overline{12}$ & 2006 & 12 & 2056 & 11 & $18 \%$ & 13 & 2236 & 12 & 204 \\
\hline
\end{tabular}

\section{GRÁFICO N $\mathbf{N}^{\circ}$}

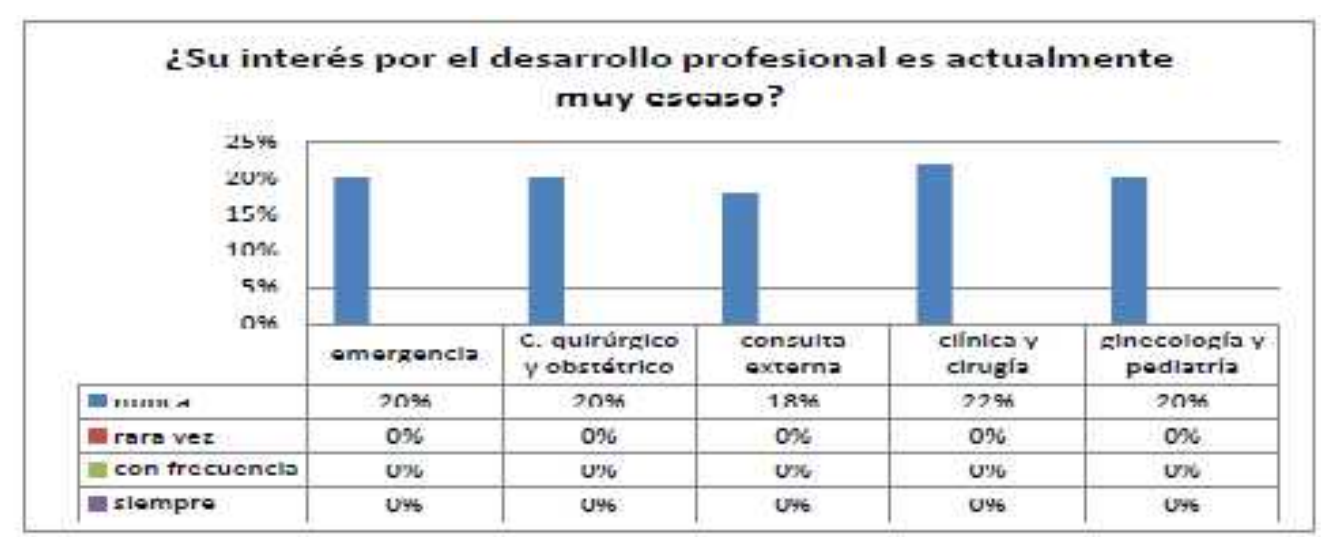

Fuente: Encuesta realizada a los profesionales de enfermería del Hospital Básico Jipijapa 


\section{ALTERACIONES FISICO SOMATICAS}

\section{CUADRO $\mathrm{N}^{\circ} 4$}

¿Considera que el trabajo que realiza, repercute en su salud personal. Padeciendo constantemente alteraciones físico somáticas?

\section{GRÁFICO $\mathbf{N}^{\circ} 4$}

\begin{tabular}{|c|c|c|c|c|c|c|c|c|c|c|}
\hline ALTCRACIONES & CMERCLNCIA & $x$ & $\begin{array}{c}\text { C. } \\
\text { QUIRÚRGICO } \\
y \\
\text { ORSTFTRICO }\end{array}$ & $x$ & $\begin{array}{c}\text { CONSULTA } \\
\text { EXIEKNA }\end{array}$ & $\%$ & $\begin{array}{c}\text { crivica } \\
\text { Y } \\
\text { CIRUGIA }\end{array}$ & $x$ & $\begin{array}{c}\text { GIVECOLOGIA } \\
\text { Y VEUIA INÍA }\end{array}$ & $x$ \\
\hline Cerzless & 2 & $7 \%$ & 1 & $2 x$ & 2 & $3 x$ & 3 & $5 \mathrm{~N}$ & 1 & $2 \%$ \\
\hline Inzomnio & 0 & $C x_{6}$ & 1 & $2 x$ & 0 & $\alpha x$ & 0 & $\mathrm{ox}$ & 0 & $0 \%$ \\
\hline $\begin{array}{l}\text { problemas } \\
\text { tligmeliven }\end{array}$ & 0 & $\mathrm{CH}$ & $u$ & ur & 0 & OS & 0 & $\mathrm{ur}$ & 2 & $v \%$ \\
\hline $\begin{array}{l}\text { Infesclones } \\
\text { trecionte: }\end{array}$ & 0 & $9 \%$ & 0 & $c x$ & 0 & $O x$ & 0 & ox & o & $0 \%$ \\
\hline iztiga cronica & 0 & $\mathrm{Cos}$ & 0 & $\infty x$ & 0 & $\infty x$ & 0 & $O x$ & 2 & $0 \%$ \\
\hline $\begin{array}{l}\text { problemas } \\
\text { musculares }\end{array}$ & $=$ & 590 & , & $=96$ & , & $79 \%$ & 4 & $7 \%$ & 3 & $5 \%$ \\
\hline $\begin{array}{l}\text { no manifiesto } \\
\text { nistruans }\end{array}$ & 1 & 1246 & 8 & 235 & , & 215 & 6 & 1066 & $s$ & 1346 \\
\hline Tnта1 & 17 & $30 \%$ & 17 & $20 \%$ & 11 & s.h & 13 & गक & 17 & $20 \%$ \\
\hline
\end{tabular}

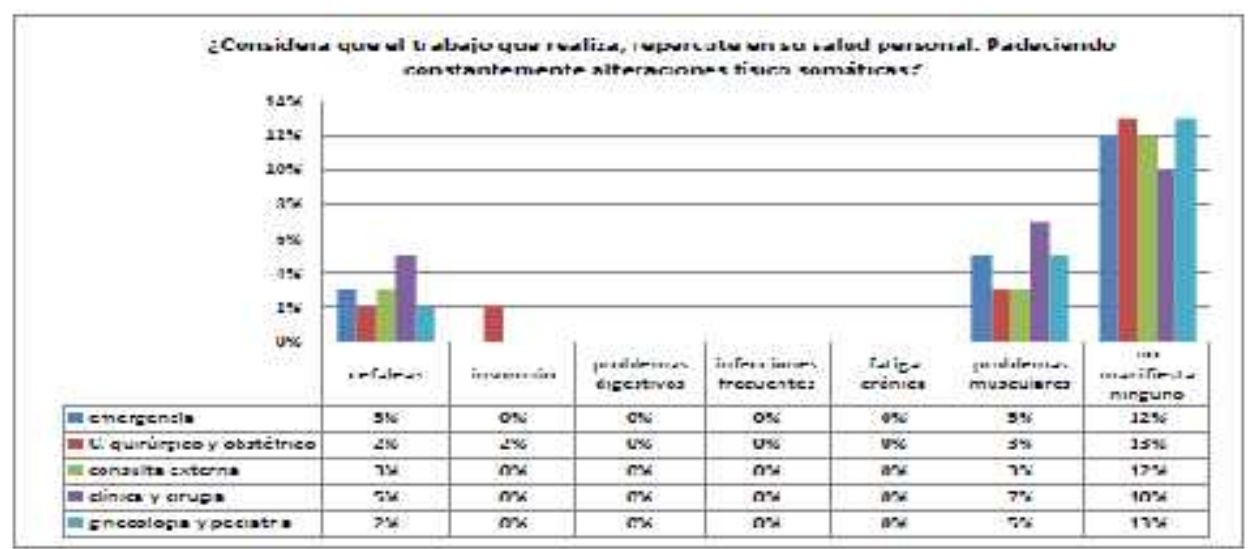


Fuente: Encuesta realizada a los profesionales de enfermería del Hospital Básico Jipijapa

\section{ESTRATEGIA ORGANIZACIONAL}

\section{CUADRO $\mathbf{N}^{\circ} 5$}

¿Las instalaciones y facilitan su trabajo y la usuarios?

\begin{tabular}{|c|c|c|c|c|c|c|c|c|c|c|}
\hline UPCIÓN & EMEKGENCLA & 40 & $\begin{array}{c}\text { C. } \\
\text { QUIRÚRGICO } \\
\mathbf{Y} \\
\text { U日SIÉIKULO }\end{array}$ & 90 & $\begin{array}{c}\text { CONSULTA } \\
\text { EXTERRA }\end{array}$ & 90 & $\begin{array}{c}\text { CLEICA } \\
\mathbf{Y} \\
\text { CIRUGIA }\end{array}$ & 40 & $\begin{array}{l}\text { GINECOLOGIA } \\
\text { Y PEDIATRIS }\end{array}$ & 90 \\
\hline numes & 0 & $7 \%$ & 0 & $0 \%$ & a & $2 \%$ & 0 & $0 \%$ & $\mathrm{c}$ & $0 \%$ \\
\hline rara vez & 0 & $2 \%$ & 0 & $0 \%$ & 0 & $2 \%$ & 0 & $0 \%$ & c & $0 \%$ \\
\hline \multicolumn{11}{|l|}{ con } \\
\hline frecuencia & 10 & $17 \%$ & 6 & $10 \%$ & 9 & $15 \%$ & 10 & $17 \%$ & 5 & $8 \%$ \\
\hline siknup & 2 & $3 \%$ & 6 & $10 \%$ & 2 & $3 \%$ & 3 & $5 \%$ & 7 & $12 \%$ \\
\hline TOTAL & 12 & $20 \%$ & 12 & $20 \%$ & 11 & $18 \%$ & 13 & $22 \%$ & 12 & $20 \%$ \\
\hline
\end{tabular}

\section{GRÁFICO $\mathbf{N}^{\circ} 5$}

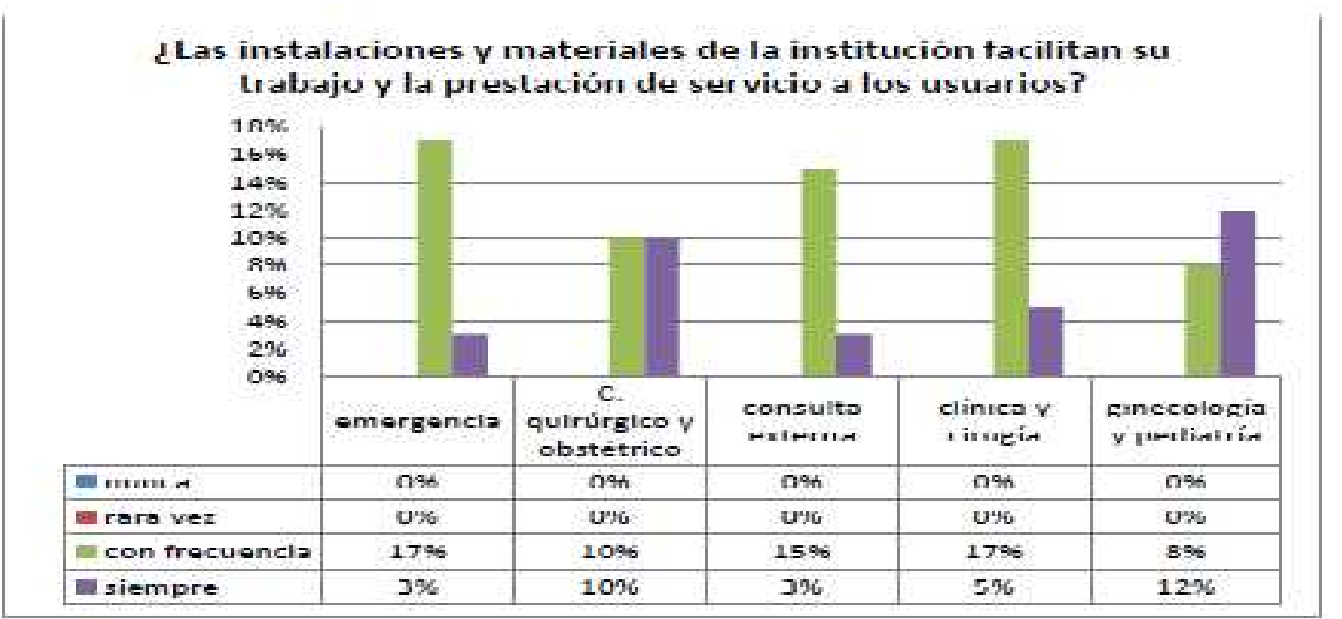

Fuente: Encuesta realizada a los profesionales de enfermería del Hospital Básico Jipijapa materiales de la Institución prestación de servicio a los 
De acuerdo a los datos obtenidos de los 60 profesionales de enfermería encuestados en el cuadro 1 grafico 1 se puede destacar de la pregunta ¿Cuando está en su trabajo se siente de mal humor? el $17 \%$ del servicio de emergencia responde que nunca y el 3\% que rara vez, del personal de centro quirúrgico y obstétrico el $18 \%$ responde que nunca y el $2 \%$ que rara vez, del personal de consulta externa el 18\% responde que nunca, en el área de cirugía el $20 \%$ responde que nuca y el $2 \%$ que rara vez, del personal de ginecología y pediatría el $18 \%$ responde que nunca y el $2 \%$ que rara vez por lo tanto el $91 \%$ de profesionales de enfermería de este estudio no presenta el síntoma de cansancio emocional pero existe un $9 \%$ que lo experimenta rara vez por lo que esto los podría predisponer a cansancio emocional. En la tabla dos grafico dos podemos decir que el $18 \%$ de los profesionales pueden no estar relacionándose correctamente con los pacientes ya que perciben rara vez la gratitud por parte de estos, lo que podría predisponer a la despersonalización y el $82 \%$ percibe una respuesta positiva con lo que determina una buena relación personal-usuario con lo que se descarta la despersonalización.

En el cuadro tres grafico tres se puede indicar que el $100 \%$ de los profesionales de enfermería tienen interés por el desarrollo profesional, por lo que se puede decir que presentan alta realización profesional. En la tabla y grafico número cuatro en cuanto a las alteraciones físico somáticas el $15 \%$ presenta cefaleas, el $23 \%$ problemas musculares mientras que el $60 \%$ no presenta alteraciones. En cuanto al cuadro y grafico cinco mencionan que los profesionales de enfermería en estudio en un porcentaje considerable sienten conformidad con las instalaciones y materiales de la institución ya que facilitan su trabajo y la prestación de servicios a los usuarios en un $77 \%$ siempre y en un $23 \%$ con frecuencia, convirtiéndose así este factor en una estrategia organizacional ayudando a prevenir estresores en el grupo de estudio.

\section{Discusión}

El cuestionario breve de burnout aplicado a los profesionales de enfermería en estudio proporciono datos relevantes y fidedignos en la cual se evidencia que el profesional de enfermería del hospital básico jipijapa presenta factores de riesgo que lo predisponen al burnout pero no padecen del síndrome presentando raras veces alteraciones físico-somáticas como cefalea, insomnio, problemas musculares lo cual comprueba que el grupo en estudio presenta estrés de nivel bajo.

El síndrome de burnout se manifiesta bajo tres componentes principales como: alto nivel de cansancio emocional, alta despersonalización en el trato y baja realización personal, del profesional de enfermería encuestado solamente el $9 \%$ presenta cansancio emocional, despersonalización el 10\% y tienen percepción alta de realización profesional el 100\% lo cual 
discrepa con las características del burnout en el que el cansancio emocional y la despersonalización son altas y la realización profesional es baja no presentando un nivel acorde al fenómeno en estudio.

Además se rescata que la institución ofrece al grupo en estudio las estrategias organizacionales proporcionando una institución con instalaciones y materiales apropiados que faciliten a los trabajadores la labor y prestación de servicio a los usuarios ayudando a prevenir estresores en el grupo de estudio.

En un estudio realizado en Costa Rica se identificó que el cansancio emocional y físico en los profesionales de enfermería se encuentran presentes, ya que factores como el estrés condicionan la aparición de los mismos, además consideran que el personal de enfermería se caracteriza por el liderazgo y la toma de decisiones sin embargo poseen poca tolerancia a estas condiciones. En cuanto al ambiente laboral donde se desenvuelven es deficiente sin embargo no revelo gran peso, pero esto puede repercutir de alguna manera en la calidad de los servicios que se brinda al usuario.

Es evidente que el profesional de enfermería cuenta con gran número de estresores que le pueden causar diversos trastornos, sin embargo es imprescindible que este personal este consciente de la responsabilidad de cumplir con calidad y calidez la atención al usuario aplicando las medidas necesarias, logrando el objetivo propuesto.

\section{Conclusiones}

De acuerdo a las características y consecuencias del burnout se concluye que el personal en estudio no padece de este fenómeno pero si presenta factores de riesgo bajo, lo cual es positivo viéndolo desde el punto de vista del estado de salud de los miembros del sistema de profesionales sanitarios en el país, ya que pueden seguir laborando con normalidad brindando atención de calidad.

A través de la investigación se determina que la mayoría de respuesta de los encuestados ha sido positiva, por lo que podemos considerar que, a nivel gerencial, los profesionales de enfermería utilizan con frecuencia estrategias individuales, grupales y organizacionales que les ayudan a prevenir o a afrontar el estrés, evitando así el síndrome de burnout

Por lo tanto es de gran importancia saber identificar los factores determinantes del sindrome de burnout y tomar las medidas correctivas antes que la salud mental de los profesionales se vea afectado. 


\section{Bibliografía}

1. Albadalejo, V. O. (2004). Sindroem de Burnout en el personal de enfermeria de un hospital de Madrid. revista Española de Salud Publica.

2. Alvarez L, F. (2000). El Sindrome de Burnout o el desgaste profesional. asociacion española de neurocirugia.

3. Bernal, L. y. (2002). Prevalencia y Factores Asociados con el Sindrome de Burnout en Enfermeria de atencion hospitalaria. DOYMA.

4. Cabrera, G. L. (2005). Sindrome de Burnout en personal de Enfermeria de un Hospital Mexicano, 11-16.

5. De la Peña, E. (2002). Sindrome de Burnout y Actitudes Disfuncionales en el Profesional de Enfermeria. Revista Meta.

6. De Pablo, C. (2007). El Sindrome de Burnout y los profesionales sanitarios. Nursing.

7. Escriba V, M. R. (2000). estresores laborales y bienestar psicologico. Rev Rol Enf, 506-511.

8. Gi- Monte, P. (2013). Sindrome de quemarse por el trabajo en profesionales de enfermeria. revista electronica intercao, 19-33.

9. Gil Monte, P. R. (2000). aproximaciones psicosociales y estudios diagnosticos sobre el sindrome de quemarse por el trabajo. psicologia del trabajo y de las organizaciones, 101-105.

10. Hernandez, J. (2003). Estres y Burnout en los profesionales de salud de los niveles primario y secundario de atencion. Revista Cubana de Salud Publica, 103-109.

11. Leiter, M. y. (2005). Six strategies for improving your relationswit whit work. San Francisco.

12. Lopez Sorianno, F. y. (2002). Prevalencia y factores asociados con el Sindrome de Burnout en enfermeria de atencion hospitalaria. Revista de Calidad Asistencial, 201-205.

13. Maslach, C. y. (1982). The Maslach Burnout Inventory y research edition . Palo Alto.

14. Noboa, M. N. (2005). Relacion entre perfil psicologico calidad de vida y estres asistencial en personal de enfermeria. Universitas Psychologica, 63-75.

15. Tous, J. (2005). Quemarse el el trabajo: El Sindrome de Burnout. Borrmart. 